\title{
Resection of a laryngeal hemangioma in an adult using an ultrasonic scalpel: A case report
}

\author{
XURUI WANG ${ }^{1,2}$, XIAODONG ZHAO ${ }^{2}$ and WEI ZHU ${ }^{1}$ \\ ${ }^{1}$ Department of Otorhinolaryngology, First Hospital of Jilin University, Changchun, Jilin 130021; \\ ${ }^{2}$ Department of Otorhinolaryngology, Jilin Province Cancer Hospital, Changchun, Jilin 130012, P.R. China
}

Received June 23, 2014; Accepted February 23, 2015

DOI: 10.3892/ol.2015.3069

\begin{abstract}
Adult laryngeal hemangioma is an extremely rare and slowly progressing vascular tumor. The present study describes the first reported case of a male with a large laryngeal hemangioma that was treated by ultrasonic scalpel. A 61-year-old male presented to our hospital with a recurrent pharyngeal foreign body sensation, without hoarseness, hemoptysis, expectoration or dyspnea. A blue-black mass was detected in the right pyriform sinus, with a morular surface and a wide pedicle positioned lateral to the right arytenoid cartilage and aryepiglottic fold under electronic laryngoscopy. Following tracheotomy under local anesthesia, right superior laryngeal artery ligation and laryngeal hemangioma resection via a lateral neck hypopharyngeal approach were performed under general anesthesia using an ultrasonic scalpel. Pathological examination verified that the tumor was a cavernous hemangioma. On day 11, subsequent to post-operative anti-inflammatory and symptomatic treatment, electronic laryngoscopy showed that the arytenoid mucosal edema had decreased and that the movement of the arytenoid was good. There was no recurrence of hemangioma during a 2-year follow-up period. Therefore, it is recommended that complete surgical resection using an ultrasonic scalpel should be considered for similar cases involving large laryngeal hemangiomas.
\end{abstract}

\section{Introduction}

Hemangiomas are the most common congenital benign tumors diagnosed, and $\sim 60 \%$ arise in the head and neck region $(1,2)$. Laryngeal hemangiomas are slowly progressing vascular tumors $(1,2)$, which are commonly diagnosed in children, but are rarer in adults. They may occur in the vocal chamber, arytenoid cartilage or aryepiglottic fold $(1,2)$. Hemangiomas occur

Correspondence to: Dr Wei Zhu, Department of Otorhinolaryngology, First Hospital of Jilin University, 71 Xinmindajie Street, Changchun, Jilin 130021, P.R. China E-mail: zhuwei167@163.com

Key words: laryngeal hemangioma, ultrasonic scalpel, video laryngoscopy rarely in the larynx, particularly in adults. Symptoms include hoarseness, dyspnea, dysphagia or a pharyngeal foreign body sensation. Due to the rarity of laryngeal hemangiomas, the exact incidence of the tumor remains unclear. In the present study, the treatment of an adult with a large laryngeal hemangioma is described.

Currently, there is no consensus regarding the treatment of hemangiomas due to their rarity. For small laryngeal hemangiomas, observation is usually sufficient. However, larger hemangiomas require treatment, and this can include surgical resection, corticosteroid injections, ethanol injections, cryosurgery, radium or gold implants, interferon treatment and laser surgery $(1,2)$. The ultrasonic scalpel has been applied in a number of head and neck surgeries and was found to significantly decrease time to hemostasis and reduce surgery duration (3); Prgomet et al (3) performed thyroidectomies, parotidectomies and tonsillectomies using the harmonic scalpel and found that the time of thyroid and parotid surgeries was reduced by $20-25 \%$ when compared with the use of conventional hemostatic techniques. In the present study, the use of an ultrasonic scalpel for the complete resection of a hemangioma via a lateral neck hypopharyngeal approach is described.

\section{Case report}

On June 7, 2011, a 61-year-old male presented to the First Hospital of Jilin University (Changchun, China) with a recurrent pharyngeal foreign body sensation, without hoarseness, hemoptysis, expectoration or dyspnea. The patient also had a history of smoking 20 cigarettes per day for the past 40 years. A video laryngoscopy was performed and a blue-black-colored mass was detected in the right pyriform sinus. The mass exhibited a morular surface and a wide pedicle positioned lateral to the right arytenoid cartilage and aryepiglottic fold (Fig. 1). The mucosae of the bilateral false vocal cords and vocal cords were smooth, and good movement of the bilateral glottis vocalis was also observed. Therefore, the patient was initially diagnosed with a laryngeal hemangioma.

Following hospitalization, a tracheotomy was performed under local anesthesia. General anesthesia was then administered to ligate the right superior laryngeal artery and resect the laryngeal hemangioma via a lateral neck hypopharyngeal approach. A $3.0 \times 2.5-\mathrm{cm}^{2}$ blue-black-colored mass was found lateral to the right arytenoid cartilage and aryepiglottic fold. 


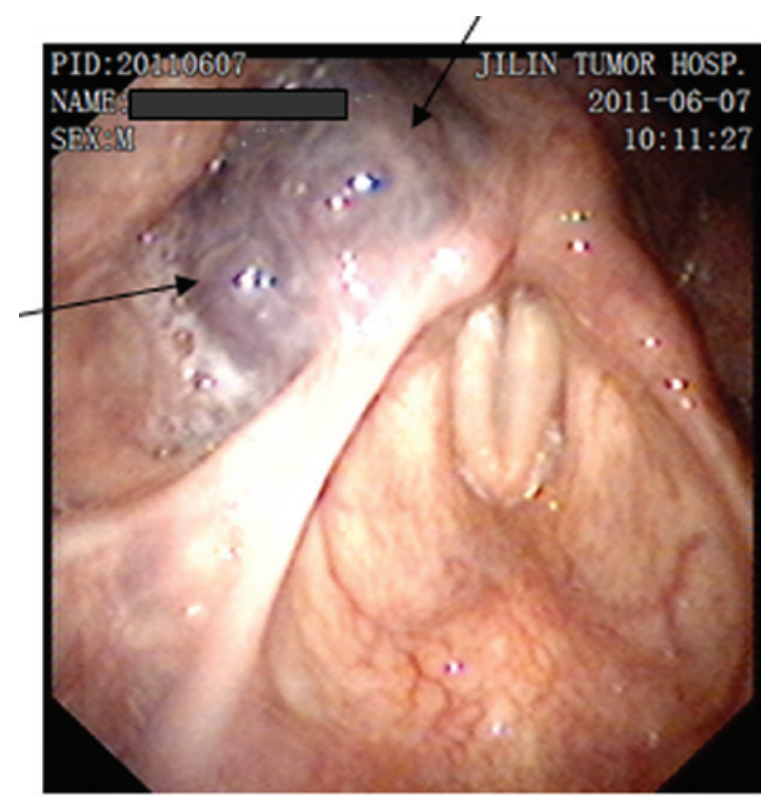

Figure 1. Video laryngoscopy was performed prior to surgery. During this procedure, a $3.0 \times 2.5-\mathrm{cm}^{2}$ blue-black-colored mass (arrows) was detected in the right pyriform sinus. The mass exhibited a morular surface and a wide pedicle positioned lateral to the right arytenoid cartilage and aryepiglottic fold.

A

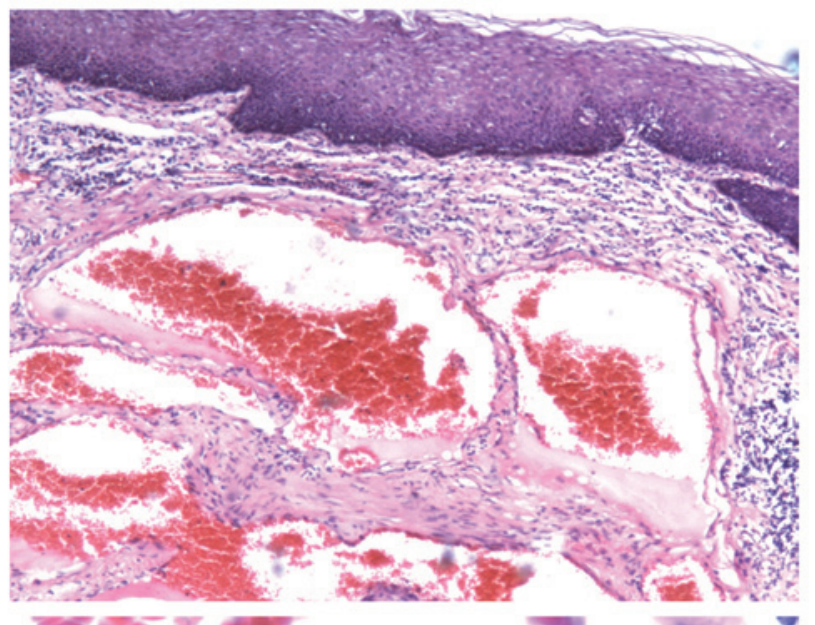

B

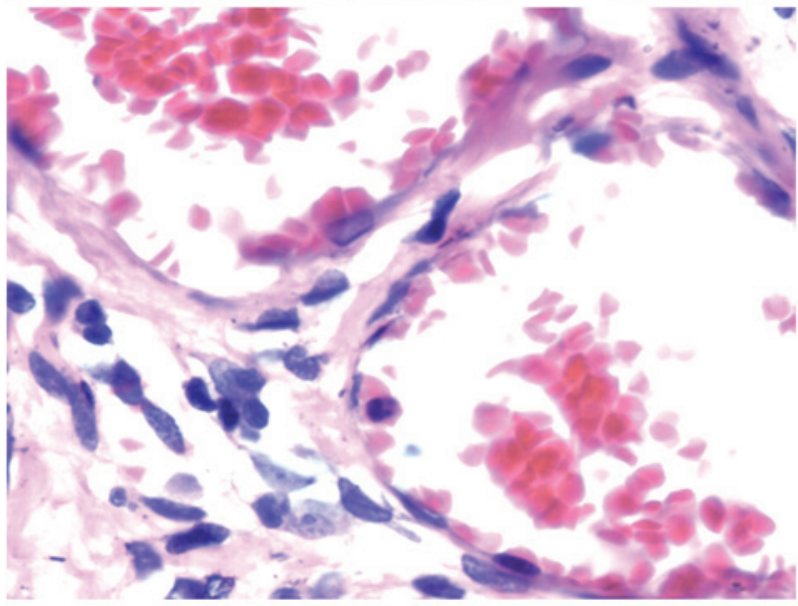

Figure 2. Hematoxylin and eosin staining of cavernous hemangioma sections. Blood vessel hyperplasia and hemangiectasis squamous mucosa were observed beneath the squamous mucosa. A phlebolith was also found in the vessel and lymphocyte infiltration was observed around the vessel. (A) $\mathrm{x} 40$ and (B) $\mathrm{x} 400$ magnification.

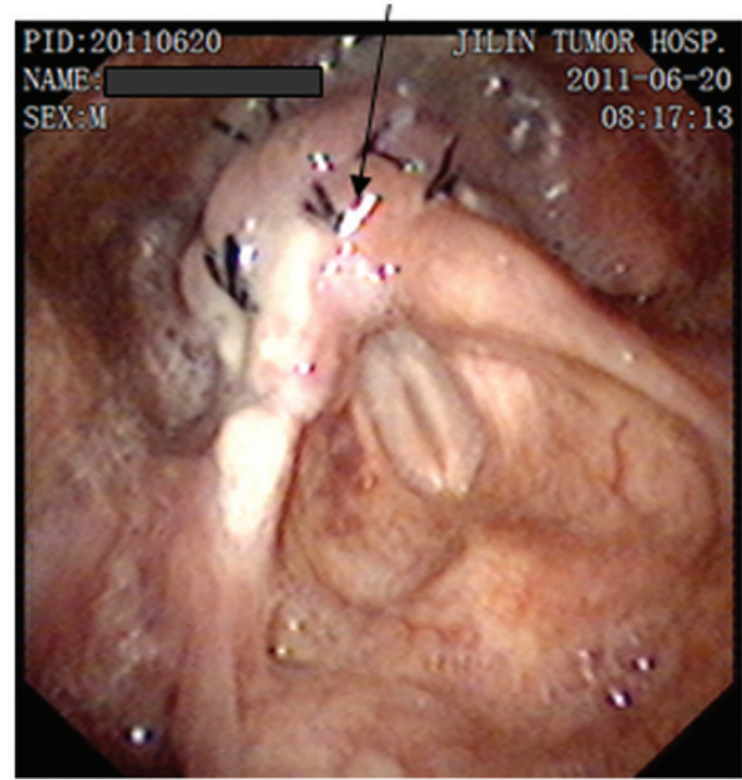

Figure 3. A second video laryngoscopy was performed on post-operative day 11 . The suture for the incision in the arytenoid cartilage mucosa (arrow) was found to be well fixed, the mucosal edema was decreased and good movement of the arytenoids was observed.

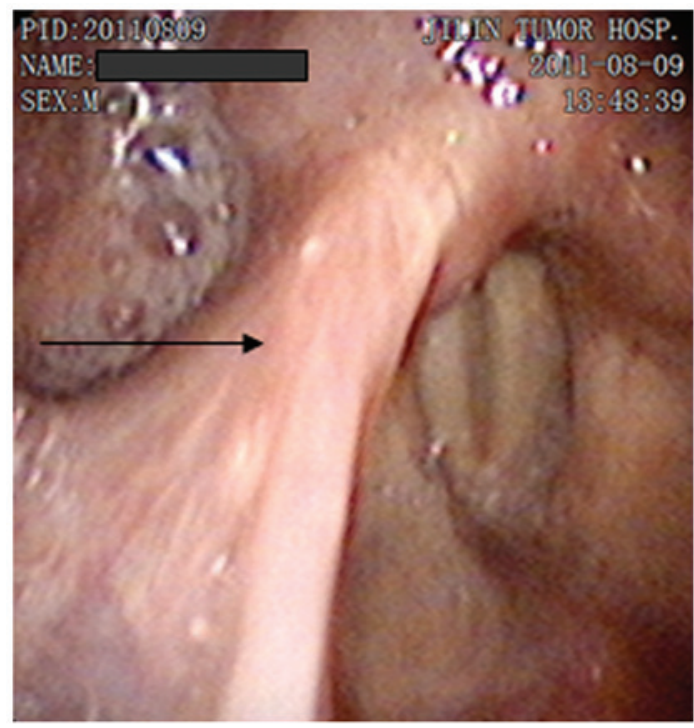

Figure 4. A third video laryngoscopy was performed at 2 months post-operatively. The suture was found to be ablated and the incision in the arytenoid cartilage was healed. A smooth surface was observed for the mucous membrane of the right pyriform sinus (arrow)

An incision was made between the normal mucosa and the margin of the hemangioma using an ultrasonic scalpel (Harmonic FOCUS ${ }^{\circledR}$ FCS9, Johnson \& Johnson, New Brunswick, NJ, USA; cutting power, $5 \mathrm{~W}$; hemostasis power, $3 \mathrm{~W}$ ). The hemangioma was separated from the normal tissue present under a shallow layer of cartilage membrane, and after narrowing the pedicle, the hemangioma was completely excised. A pathological examination detected blood vessel hyperplasia and hemangiectasis squamous mucosa beneath the squamous mucosa. In addition, a phlebolith was present in 
the vessel and lymphocyte infiltration was observed around the vessel. Thus, the diagnosis of a cavernous hemangioma was confirmed (Fig. 2).

Post-operatively, anti-inflammatory and symptomatic treatment was administered. On day 11, a video laryngoscopy showed that the suture of the arytenoid cartilage mucosa incision was well fixed, the mucosal edema was reduced and good movement of the arytenoid was observed (Fig. 3). Therefore, the nasogastric tube and tracheal cannula were removed. At 2 months post-operatively, an additional video laryngoscopy showed that the suture was ablated and the arytenoid cartilage mucosa incision had healed. Furthermore, no recurrence of the hemangioma was detected during a 2-year follow-up period (Fig. 4).

The patient provided informed consent for the present study, and the study protocol was approved by the Ethics Committee of the First Hospital of Jilin University.

\section{Discussion}

Laryngeal hemangiomas have been reported in the vocal chamber, arytenoid cartilage and aryepiglottic fold, and rarely occur in the larynx $(1,2)$. Symptoms include hoarseness, dyspnea, dysphagia or a pharyngeal foreign body sensation. In the present study, an adult experiencing only a recurrent pharyngeal foreign body sensation was diagnosed with a laryngeal hemangioma in the absence of other symptoms.

In previous studies, computed tomography (CT) and magnetic resonance imaging (MRI) have been found to be useful for defining the form, size and anatomical association of hemangiomas. Moreover, Chetty et al (4) demonstrated that CT with contrast facilitated the detection of subglottic hemangiomas, and also showed the degree of airway narrowing. Correspondingly, it was suggested that CT could potentially differentiate vascular rings and subglottic lesions, as well as other extrinsic masses involving the neck and upper mediastinum. Alternatively, MRI was found to provide excellent differentiation of fat and muscle, thereby allowing the depth of vascular malformation extensions and delineation of tumor from normal tissue to be determined. For example, MRI results from a study by Gelbert et al (5) suggested that MRI is the best adjunctive indication for capillary-venous hemangiomas. In the present case, video laryngoscopy identified a blue-black-colored mass located in the right pyriform sinus. The mass exhibited a morular surface and a wide pedicle positioned lateral to the right arytenoid cartilage and aryepiglottic fold. Similarly, Huang et al (6) reported a lobulated, dark-red tumor occupying the aerodigestive tract that was diagnosed as a large laryngeal hemangioma according to video imaging obtained during a laryngoscopy. Taken together, these results suggest that the combined use of CT, MRI and laryngoscopy will facilitate the delineation and diagnosis of laryngeal hemangiomas characterized by deep extensions.

For hemangiomas, a pathological examination is typically required to form a definitive diagnosis. Currently, the histopathology of hemangiomas is classified into three types according to vessel size and wall thickness. These types consist of a capillary type, a cavernous type and a mixed type (7). The cavernous type is most commonly observed, and it involves an expanded vessel with a thin wall (7). In the present case, blood vessel hyperplasia and hemangiectasis squamous mucosa were present beneath the squamous mucosa, and a phlebolith was present in the vessel. Lymphocyte infiltration of the vessel region was also observed. Correspondingly, the hemangioma examined was considered cavernous.

Due to the small number of cases reported involving laryngeal hemangiomas, there is currently no consensus regarding treatment $(1,2,4-7)$. For small or asymptomatic laryngeal hemangiomas, close observation has generally been found to be sufficient. However, large hemangiomas require therapeutic treatment in order to prevent the ulceration, necrosis or infection of vital organs $(1,2)$. To date, several treatment options have been reported, and these include surgical resection, corticosteroid injections, ethanol injections, cryosurgery, radium or gold implants, interferon treatments and laser surgery (2). In a study by Lucioni et al (8), 6 consecutive cases of adult laryngeal hemangiomas were treated with $\mathrm{CO}_{2}$ laser microsurgery alone. Of these, only 1 patient presented with limited disease control in the retro-cricoid and arytenoids after a 20-month follow-up period. In another study, Kawakami et al (9) treated a giant hemangioma present in the larynx of an adult using coagulation induced by a potassium titanyl phosphate laser via a tracheotomy. For this patient, the hemangioma did not progress up to 9 months after the treatment. Huang et al (6) also reported that radiation therapy involving a total dose of 40 Gy administered in 20 fractions successfully relieved symptoms and was accompanied by moderate tumor regression in a patient suffering from a life-threatening laryngeal hemangioma involving the pharynx and parapharyngeal space (6). The study further suggested that radiation therapy may be a treatment approach to consider for intractable and function-threatening laryngeal hemangiomas. In the present case, the right superior laryngeal artery was ligated to avoid hemorrhage, and a lateral neck hypopharyngeal approach was used to reduce the potential for injury to the larynx. To the best of our knowledge, this represents the first report of an ultrasonic scalpel used for the complete resection of a hemangioma. In addition, only $50 \mathrm{ml}$ of blood loss occurred during surgery, and recurrence of the hemangioma was not observed during 2 years of follow-up. In another study, an ultrasonic scalpel was applied to the treatment of periorbital hemangiomas, and this was found to be a safe and effective alternative to pharmacological therapy (10). Moreover, in a study of 81 children affected by immature hemangiomas, ultrasonic scalpel dissection was found to significantly contribute to surgical outcome, was a safe treatment option and was associated with a shorter surgical duration (11). Taken together, these results suggest that the use of an ultrasonic scalpel represents a safe, effective and less invasive method for the treatment of large laryngeal hemangiomas. Based on the limitations of the present study, which included the use of data from a single institution and a small sample size, additional multicenter, randomized controlled trials are required in order to confirm whether the use of ultrasonic scalpel resection represents an effective treatment for laryngeal hemangiomas.

The present study reported the case of a male with a large laryngeal hemangioma. It is recommended that a complete surgical resection using an ultrasonic scalpel via a lateral neck hypopharyngeal approach should be considered for similar cases. 


\section{References}

1. Martins RH, Lima Neto AC, Semenzate G and Lapate R: Laryngeal hemangioma. Braz J Otorhinolaryngol 72: 574, 2006.

2. Zheng JW, Zhou Q, Yang XJ, et al: Treatment guideline for hemangiomas and vascular malformations of the head and neck. Head Neck 32: 1088-1098, 2010.

3. Prgomet D, Janjanin S, Bilić M, Prstacić R, Kovac L, Rudes M and Katić V: A prospective observational study of 363 cases operated with three different harmonic scalpels. Eur Arch Otorhinolaryngol 266: 1965-1970, 2009.

4. Chetty A, Mischler E and Gregg D: Diagnosis of subglottic hemangioma by chest CT. Pediatr Pulmonol 23: 464-467, 1997.

5. GelbertF, Riche MC, Reizine D, et al: MR imaging of head and neck vascular malformations. J Magn Reson Imaging 1: 579-584, 1991.

6. Huang CM, Lee KW and Huang CJ: Radiation therapy for life-threatening huge laryngeal hemangioma involving pharynx and parapharyngeal space. Head Neck 35: E98-E101, 2013.
7. Yang GZ, Li J and Jin H: Giant mesenteric hemangioma of cavernous and venous mixed type: a rare case report. BMC Surg 13: 50, 2013

8. Lucioni M, Marioni G, Della Libera D and Rizzotto G: Adult laryngeal hemangioma $\mathrm{CO}_{2}$ laser excision. A single institution 3-year experience (Vittorio Veneto 2001-2003). Acta Otolaryngol 126: 621-626, 2006.

9. Kawakami M, Hayashi I, Yoshimura K, Ichihara K, Nishikawa S and Ichihara T: Adult giant hemangioma of the larynx: a case report. Auris Nasus Larynx 33: 479-482, 2006.

10. Claude O, Picard A, O'Sullivan N, et al: Use of ultrasonic dissection in the early surgical management of periorbital haemangiomas. J Plast Reconstr Aesthet Surg 61: 1479-1485, 2008.

11. Picard A, Soupre V, Diner PA, Buis J, Goga D and Vazquez MP: Early surgery of immature hemangiomas with the aid of an ultrasonic scalpel. Apropos of 81 cases. Rev Stomatol Chir Maxillofac 103: 10-21, 2002 (In French). 